\title{
Correction
}

\section{Rationality of Moduli Spaces of Stable Bundles}

\author{
P. E. Newstead
}

Department of Pure Mathematics, University of Liverpool, P.O. Box 147, Liverpool L69 3BX, UK

Math. Ann. 215, 251-268 (1975)

I am grateful to S. Ramanan for pointing out to me an error in my paper [1]. The error occurs on lines 17,18 of p. 257 , where it is assumed that $n g-d$ is coprime to $d$; this is of course false if $(g, d) \neq 1$. Since the only restrictions on $n$ and $d$ at this point are

$$
(n, d)=1, n(g-1)<d<n g,
$$

this invalidates the proof of the proposition of [1] and hence also that of the main theorem.

There seems to be no simple way of avoiding this problem, but the methods of [1] do give some positive results, as we shall now show. We adopt the notations and conventions of [1] throughout; in particular $L$ is a line bundle of degree $d$ over the complete non-singular algebraic curve $X$ of genus $g \geqq 2$, and $S_{n, L}(X)$ is the moduli space of stable bundles of rank $n$ and determinant $L$ over $X$. Note that, up to isomorphism, $S_{n, L}(X)$ depends only on the residue class of $d$ modulo $n$; moreover, if $L^{*}$ denotes the dual of $L$, then $S_{n, L}(X)$ is isomorphic to $S_{n, L}(X)$. For any bundle $E$ over $S \times X$ and any $s \in S$, we write $E_{s}$ for the bundle over $X$ obtained by restricting $E$ to $\{s\} \times X$.

Definition. A good $(n, L)$-family parametrised by a variety $S$ is a bundle $E$ of rank $n$ over $S \times X$ such that

(i) $\operatorname{dim} S=\left(n^{2}-1\right)(g-1)$;

(ii) for all $s, t \in S, E_{s} \cong E_{t}$ if and only if $s=t$;

(iii) for all $s \in S, E_{s}$ is stable and $\operatorname{det} E_{s} \cong L$;

(iv) for all $s \in S, H^{1}\left(X ; E_{s}\right)=0$;

(v) for all $s \in S$, the infinitesimal deformation map of $E$ at $s$ is injective.

Remark 1 . If $(n, d)=1$, there exists a bundle $U$ over $S_{n, L}(X) \times X$ with the obvious universal property. We shall say that the pair $(n, L)$ is good if $S_{n, L}(X)$ is a rational variety and there exists a Zariski-open subset $T$ of $S_{n, L}(X)$ such that $U \mid T \times X$ is a good $(n, L)$-family. Note that $S_{1, L}(X)$ consists of a single point; it therefore follows trivially from Riemann-Roch that $(1, L)$ is good if $d \geqq 2 g-1$. [If $n \geqq 2$ and $d>n(g-1)$, one can show that there exists a Zariski-open subset $T$ of $S_{n, L}(X)$ such that $U \mid T \times X$ is a good $(n, L)$-family, but we shall not need to use this fact.] 
Remark 2. The argument in the first paragraph of [1, Sect. 4] shows that, if there exists a good $(n, L)$-family parametrised by $S$, then $S$ is birationally equivalent to $S_{n, L}(X)$; if $S$ is a rational variety, it follows that $(n, L)$ is good. Note in particular that, by [2, Theorem 2$]$, a good ( $n, L)$-family can exist only if $(n, d)=1$.

The arguments of $[1$, Sects. 3,4$]$ now give

Proposition 1. Suppose that $n(g-1)<d<n g$ and that there exists a good $(n g-d, L)$ family $E^{\prime}$ parametrised by $T$. Then there exists a good $(n, L)$-family $E$ parametrised by a Zariski-open subset $S$ of $T \times k^{p}$ for a suitable integer $p$; moreover one can choose $S$ so that, for all $s \in S$, the sections of $E_{s}$ generate a trivial subbundle of $E_{s}$.

Corollary. Suppose that $n(g-1)<d<n g$, let $K$ denote the canonical line bundle over $X$, and let $M$ be a line bundle of degree 1 over $X$ such that $H^{0}(X ; M) \neq 0$. Suppose further that either $(n g-d, L)$ or $\left(d-n(g-1), L^{*} \otimes K^{n} \otimes M^{n}\right)$ is good. Then $(n, L)$ is good.

Proof. In the first case, we apply the proposition with $T$ as in Remark 1 and $E^{\prime}=U \mid T \times X$; the result then follows from Remark 2 . In the second case, the proposition gives us a good $\left(n, L^{*} \otimes K^{n} \otimes M^{n}\right)$-family $E$ parametrised by a rational variety $S$; moreover, from the last part of the proposition,

$H^{0}\left(X ; E_{s} \otimes M^{*}\right)=0$ for all $s \in S$.

It follows easily that $E^{*} \otimes p_{X}^{*}(K \otimes M)$ is a good $(n, L)$-family; hence $(n, L)$ is good by Remark 2.

This corollary allows us to prove that $S_{n, \mathbf{L}}(X)$ is rational for certain values of $g, n, d$. In particular we have

Proposition 2. $S_{n, L}(X)$ is rational in the following cases:

(a) $d \equiv \pm 1 \bmod n$;

(b) $(n, d)=1$ and $g$ is a prime power;

(c) $(n, d)=1$ and the sum of the $t$ wo smallest distinct prime factors of $g$ is greater than $n$.

Proof. The proposition is trivial when $n=1$; it is therefore sufficient to prove it for

$$
n \geqq 2, n(g-1)<d<n g .
$$

In this case (a) follows at once from the corollary and the fact that $\left(1, L^{\prime}\right)$ is good if $\operatorname{deg} L^{\prime} \geqq 2 g-1$; indeed we have the stronger result that $(n, L)$ is good.

For (b) and (c) we again assume $(*)$ and prove that $(n, L)$ is good by induction on $n$. The essential point is that the hypotheses imply that either $(n g-d, d)=1$ or $(d-n(g-1), n(2 g-1)-d)=1$; the inductive step now follows easily from the corollary.

Remark. The Corollary to Proposition 1 applies in many other cases (for example $d \equiv \pm 2, g$ odd); the simplest case to which our results do not apply is whan $n=5$, $d \equiv 2$ or 3 and $g$ is divisible by 6 .

\section{References}

1. Newstead, P.E. : Rationality of moduli spaces of stable bundles. Math. Ann. 215, 251-268 (1975)

2. Ramanan, S.: The moduli spaces of vector bundles over an algebraic curve. Math. Ann. 200, 69-84 (1973) 